\title{
Substantial subpial cortical demyelination in progressive multiple sclerosis: have we underestimated the extent of cortical pathology?
}

\author{
Lauren Griffiths ${ }^{1}$, Richard Reynolds ${ }^{2}$, Rhian Evans ${ }^{1}$, Ryan J. Bevan ${ }^{1}$, Mark I. Rees ${ }^{1,3}$, Djordje Gveric ${ }^{2}$, \\ James W. Neal' ${ }^{1}$, Owain W. Howell ${ }^{1,2}$ \\ ${ }^{1}$ Institute of Life Sciences, Swansea University Medical School, Swansea University, Swansea SA2 8PP, UK. \\ ${ }^{2}$ Department of Brain Sciences, Faculty of Medicine, Imperial College London, London W5 3EH, UK. \\ ${ }^{3}$ Faculty of Medicine and Health, University of Sydney, Sydney, New South Wales NSW 2006, Australia.
}

Correspondence to: Dr. Owain W. Howell, Institute of Life Sciences, Swansea University Medical School, Swansea University, Singleton Park, Swansea, SA2 8PP, UK. E-mail: o.w.howell@swansea.ac.uk

\begin{abstract}
How to cite this article: Griffiths L, Reynolds R, Evans R, Bevan RJ, Rees MI, Gveric D, Neal JW, Howell OW. Substantial subpial cortical demyelination in progressive multiple sclerosis: have we underestimated the extent of cortical pathology? Neuroimmuno/ Neuroinflammation 2020;7:51-67. http://dx.doi.org/10.20517/2347-8659.2019.21
\end{abstract}

Received: 13 Dec 2019 First Decision: 21 Jan 2020 Revised: 14 Feb 2020 Accepted: 27 Feb 2020 Published: 21 Mar 2020

Science Editor: Roberta Magliozzi Copy Editor: Jing-Wen Zhang Production Editor: Jing Yu

\begin{abstract}
Aim: Multiple sclerosis (MS) is an inflammatory demyelinating and neurodegenerative disease. Much of the complex symptomatology relates to pathology outside the classic white matter plaque, whereby lesions of the cortical grey matter, which are difficult to resolve by conventional clinical imaging, are in part predictive of outcome. We investigated the extent of grey matter pathology in whole coronal macrosections to reassess the contribution of cortical pathology to total demyelinating lesion area in progressive MS.
\end{abstract}

Methods: Twenty-two cases of progressive MS were prepared as whole bi-hemispheric macrosections for histology, immunostaining and quantitative analysis of lesion number and relative area, leptomeningeal inflammation and microglial/macrophage activation.

Results: Cortical grey matter demyelination was seen in all cases, which was more extensive than in white and deep grey matter (hippocampus, thalamus and basal ganglia) and accounted for $0.8 \%-60.2 \%$ of the entire measurable cortical ribbon. The pattern of cortical grey matter demyelination was predominantly subpial (mean $90.9 \%$, range $60 \%-100 \%$, of total cortical grey matter lesion area) and cases with the largest areas of subpial cortical lesions had more and larger deep grey matter lesions, greater numbers of activated microglia/macrophages, both in lesions as well as in normal cortical grey matter, together with elevated leptomeningeal inflammation and lymphoid-like

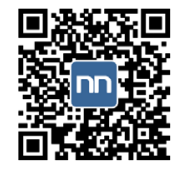


structures. White matter lesion area was unchanged when compared with the progressive MS cases with little subpial cortical demyelination.

Conclusion: Analysis of whole coronal macrosections reveals cortical demyelination is more extensive than reported by conventional histological methods. Cases of progressive MS with substantial subpial cortical demyelination that is independent of underlying white matter lesion area support the implications that these lesions may in-part arise through different pathogenetic mechanisms. Biomarkers and/or imaging correlates of this subpial pathology are required if we are to fully comprehend the clinical disease process.

Keywords: Leptomeninges, B cell follicle, microglia activation, normal appearing grey matter.

\section{INTRODUCTION}

Neocortical grey matter demyelination, microglial activation and neurodegeneration, which may in-part be driven by inflammation of the overlying leptomeninges, are important pathological processes influencing the clinical severity and outcome of multiple sclerosis $(\mathrm{MS})^{[1]}$. No biomarker or widely available imaging technology can fully report the extent of neocortical pathology, which is essential for optimal disease management.

Neocortical and cerebellar cortical pathology is evident from the earliest stages of $\mathrm{MS}^{[2-5]}$. It is a pathological hallmark of progressive $\mathrm{MS}^{[1,2]}$ and closely associates with clinical severity at all stages ${ }^{[6]}$. For example, the extent of cortical pathology is better at predicting disease outcome than white matter pathology ${ }^{[7]}$. Radiological imaging of grey matter and cortical atrophy are predictive of the conversion to clinically definite $\mathrm{MS}^{[8-10]}$ or the risk of transitioning to the progressive phase and disability ${ }^{[7,11]}$. Nevertheless, even high fidelity, non-routine, imaging technologies fail to identify lesions of the most superficial cortical layers, whilst neuropathological assessment of standard size tissue blocks often underestimates the extent of cortical lesions, which can occupy the cortical ribbon over multiple contiguous gyri and sulci $\mathrm{i}^{[2,12]}$.

Lesions of the superficial layers of the neocortex (subpial cortical grey matter lesions) are more numerous and sometimes associate topographically with leptomeningeal foci of immune cell aggregates that resemble lymphoid-like structures (alternatively termed ectopic B cell follicle structures) seen in other autoimmune, chronic inflammatory and infectious diseases ${ }^{[13,14]}$. Progressive MS cases with leptomeningeal lymphoidlike structures exhibit a gradient of cortical tissue damage extending away from the pial surface, suggestive of factor(s) in the CSF that promote underlying cortical inflammation and injury ${ }^{[15-17]}$, possibly through the activation of microglia. The degree of leptomeningeal inflammation correlates with the extent of cortical demyelination, neurodegeneration and microglial activation in acute and progressive stages ${ }^{[4,13,18,19]}$, whilst white matter lesion area is not changed. These findings suggest that subpial cortical lesions may arise through partly independent mechanisms compared with lesions located further from the CSF-filled spaces of the pia or ventricular lumen ${ }^{[17]}$.

The study of whole bi-hemispheric coronal macrosections, although technically challenging, can reveal hitherto undisclosed aspects of MS pathology and better report the extent of global pathology. We analysed the distribution and histological characteristics of cortical grey matter, white matter and deep grey matter lesions, together with inflammation of the brain and overlying leptomeninges, using whole brain coronal sections from 22 cases of progressive MS. We demonstrate the sometimes surprising extent of subpial cortical grey matter demyelinating pathology that can be seen and highlight the association between subpial and periventricular grey matter lesions, the dissociation between subpial lesion load and white matter lesion area, and confirm the close relationship between leptomeningeal inflammation and subpial 
Table 1. Cases and sections used in this study

\begin{tabular}{lllccll}
\hline Case & Sections analysed & Sex & $\begin{array}{c}\text { Age at death } \\
\text { (years) }\end{array}$ & $\begin{array}{c}\text { Disease } \\
\text { duration (years) }\end{array}$ & MS type & \multicolumn{1}{c}{ Cause of death } \\
\hline MS202 & A2, P1 & F & 58 & 23 & SPMS & Pulmonary embolism \\
MS204 & A3, P1, P3 & M & 58 & 19 & SPMS & Leukaemia and MS \\
MS212 & A3, P1, P3 & F & 47 & 29 & SPMS & Multiple sclerosis/bronchopneumonia \\
MS214 & P1 & F & 51 & 31 & SPMS & Multiple sclerosis \\
MS217 & A3, P1, P3 & F & 57 & 15 & SPMS & Suicide \\
MS223 & A2 & F & 45 & 2 & SPMS & Multiple sclerosis/bronchopneumonia \\
MS224 & A2, P1, P3 & F & 59 & 33 & SPMS & Multiple sclerosis/bronchopneumonia \\
MS226 & A2, P3 & M & 64 & 27 & SPMS & Multiple sclerosis/pneumonia \\
MS253 & A3, P1, P3 & F & 37 & 16 & SPMS & Multiple sclerosis/pulmonary embolism \\
MS257 & A2, P1, P3 & F & 49 & 22 & SPMS & Aspiration pneumonia \\
MS258 & A2, P1, P3 & M & 46 & 20 & SPMS & Multiple sclerosis \\
MS278 & A3, P1, P3 & M & 30 & 21 & SPMS & Pneumonia \\
MS293 & P1, P3 & F & 53 & 18 & SPMS & Multiple sclerosis \\
MS295 & P1, P3 & F & 71 & 15 & SPMS & Bronchopneumonia \\
MS323 & A4, P1 & F & 62 & 31 & SPMS & Multiple sclerosis/sepsis \\
MS336 & A2, P1, P3 & F & 57 & 27 & SPMS & Multiple sclerosis/resp failure \\
MS344 & P1 & F & 57 & 15 & SPMS & Multiple sclerosis/septicaemia \\
MS360 & A2, P1, P3 & M & 55 & 40 & SPMS & Multiple sclerosis \\
MS361 & P1 & F & 60 & 34 & SPMS & Multiple sclerosis \\
MS366 & P1 & F & 61 & 19 & SPMS & Multiple sclerosis/bronchopneumonia \\
MS387 & P3 & F & 43 & 11 & SPMS & Multiple sclerosis \\
MS395 & A3, P3 & M & 63 & 26 & SPMS & Multiple sclerosis/chest infection \\
\hline
\end{tabular}

List of cases, number of coronal planes analysed, disease course and principal cause of death. Sex (Female/Male), age of death and disease duration reported in years and disease type (secondary progressive MS). MS: multiple sclerosis; SPMS: secondary progressive MS

cortical lesion pathology. This data support efforts to develop brain imaging and biomarker technologies for the identification of subpial grey matter lesions to improve disease prediction and monitoring.

\section{METHODS}

\section{Post-mortem cohort}

Formalin fixed, whole brains $[n=22$; median age 57 years (range 30-71 years), median disease duration 21.5 years $(2-40$ years $)$, female $=17]$ were available from clinically and neuropathologically validated cases of secondary progressive MS (see Table 1 for details). All cases were provided by the UK Multiple Sclerosis Society Tissue Bank, Imperial College London, with appropriate research ethics approval (08/ MRE09/31+5). Case selection was based on availability of whole brains with well-preserved leptomeninges and accompanying detailed clinical and neuropathology summaries, collected between February 2004 and December 2008. Some of these cases have previously been reported ${ }^{[18]}$, but all analysis and data presented here are unique to this manuscript.

Individual progressive MS brains were dissected into 1-cm-thick coronal sections, cut in an anterior direction from the mammillary bodies as coronal bi-hemispheric sections (A1, A2 and A3), or posteriorly from the mammillary bodies towards the occipital lobe (sections P1, P2 and P3) such that each coronal section contained several different cyto-architectonic areas [Figure 1]. For example, coronal section A3 includes frontal cortex and poles of the temporal gyrus; coronal section P1 includes motor and somatosensory cortex, thalamus and anterior hippocampus; and coronal section P3 includes parietal and occipital lobe and occipital horn of the lateral ventricle. Areas of interest for comparison were subdivided into: (1) cortical (neocortex); (2) white matter; and (3) hippocampus and deep grey matter [comprising caudate, pallidum (interna and externa), putamen, thalamus, hypothalamus and hippocampus and dentate gyrus]. 


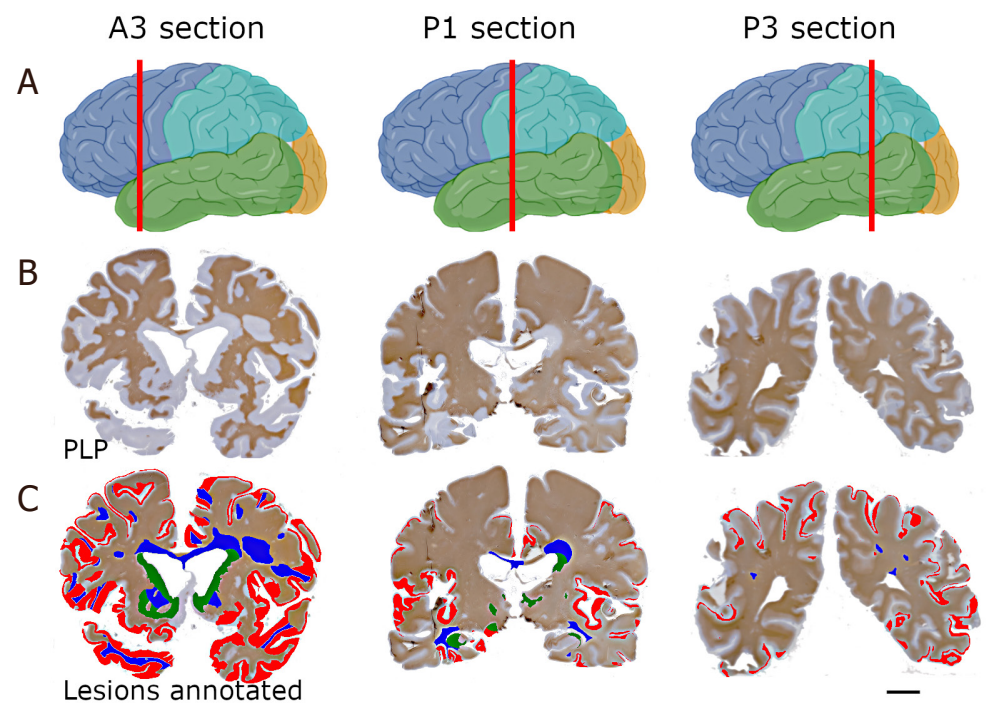

Figure 1. Study setup and sampling protocol. Whole coronal tissue slabs sectioned along coronal planes $A 3, \mathrm{P} 1$ and $\mathrm{P} 3$ were prepared at three levels from immersion fixed brains (A) (created with Biorender.com). Histology and immunohistochemistry (anti-PLP immunostaining) revealed tissue anatomy and areas of frank demyelination (B) (MS278 section A3, MS202 section P1 and MS360 section P3). Stained and mounted tissue sections were digitised and colour masks depicting demyelinating lesions involving the neocortical grey matter (red colour mask), deep grey matter and hippocampus (green colour mask) and white matter (blue colour mask) were annotated for lesion quantification (C). (B, C) Scale bar $=2 \mathrm{~cm}$. PLP: proteolipid protein

\section{Tissue preparation, staining and characterising demyelinated lesions}

Whole coronal tissue sections were processed under vacuum and paraffin wax embedded prior to sectioning at $10 \mu \mathrm{m}$ onto 3-Aminopropyltriethoxysilane-silane (Sigma, UK) coated glass slides using a Reicheart-Jung tetrander microtome. Following dewaxing and rehydration, coronal sections from each case were stained with luxol fast blue (LFB) and cresyl violet to identify anatomical landmarks (grey and white matter) and to facilitate the assessment of total cortical, hippocampal and deep grey matter and white matter areas. Subsequent sections were immunostained with anti-proteolipid protein (PLP) and anti-CD68 primary antisera [Table 2] and revealed by sequential anti-species specific biotinylated secondary and avidin-biotin complex (Vector Labs. Ltd.) horse-radish peroxidase conjugated tertiary detection reagent with ImmPACT DAB as the reporter chromogen (Vector). All slides were counterstained, dehydrated, cleared in xylene and DePeX mounted under glass coverslip (Ted Pella Inc. USA).

Cortical grey matter lesions (GMLs), revealed by anti-PLP immunohistochemistry, were grouped as Type I (leukocortical), Type II (intracortical and not contacting the pia or grey/white matter boundary), or Type III (extending from the pia, sometimes involving the entire breadth of the cortex and stopping at the grey/white matter boundary (Bo Type IV cortical GML ${ }^{[20]}$ ). Areas of sparse and patchy anti-PLP immunostaining, which reflect incomplete demyelination and/or remyelination, but are notably different to the most "normal" grey matter, were labelled as partially de/re-myelinated grey matter ${ }^{[4]}$.

White matter lesions (WMLs) were separately characterised as active inflammatory (confluent in CD68+ microglia/macrophages), chronic active (activated microglia/macrophages restricted to the lesion edge containing PLP+ and/or LFB+ myelin breakdown products) and chronic inactive lesions (few activated microglia/macrophages at the lesion edge without evidence of recent myelin phagocytosis). Additional areas of putative remyelinating/shadow plaque (by PLP+ myelin internodes and a classic LFB+ shadow appearance) were identified but not quantified. Demyelinating lesions of the hippocampus and deep grey matter were identified based on a clear loss of LFB and/or PLP+ immunostain and were characterised as active, chronic active or chronic inactive inflammatory demyelinating lesions ${ }^{[2]}$. 
Table 2. List of primary antibodies used

\begin{tabular}{lllc}
\hline Antibody & Dilution & \multicolumn{1}{c}{ Target antigen } & Company and product details \\
\hline Anti-CD68 & $1: 400$ & CD68 (macrosialin) & Dako; clone kp1 \\
Anti-CD20 & $1: 125$ & CD20 & Dako; clone I26 \\
Anti-PLP & $1: 1500$ & Myelin proteolipid protein & BioRad; clone plpc1 \\
\hline
\end{tabular}

Primary antibody, working dilution, principal target and product details. PLP: proteolipid protein

\section{Slide digitisation, lesion masks and quantitative analysis}

Individual coronal sections were reviewed using an Olympus SZ60 stereo microscope 0.1-10 $\times$ magnification) and a Zeiss AxioImager Z1 (40-400 $\times$ magnification) to identify normal and pathological regions of interest in each slide, which were marked on A4-sized print-outs of the same slide captured using a conventional document scanner (HP Scanjet 300) at 1200 dpi.

Quantifying the number and area of cortical, white matter and hippocampus and deep grey matter lesions The scanned images were used to guide our tracing of white and grey matter areas (from the LFB stained section), and areas of cortical, white matter and hippocampus and deep grey matter lesions (PLP+ slide) as colour-mask overlays using GNU image manipulation software (GIMP 2.10; see Figure 1). The modified high-resolution TIFF images were analysed in ImageJ (https://imagej.net/Fuiji/Downloads) to record the total number and area $\left(\mathrm{mm}^{2}\right)$ of cortical grey matter, white matter and hippocampus and deep grey GML area per section, per case. We defined the maximum extent of a Type III lesion for lesion counting as an area of complete demyelination that extended over a maximum of two entire sulci and gyri, as some Type III lesions extended across three or more gyri or involved the entire superficial cortical grey matter in a single hemisphere. Therefore, our Type III lesion count represents individual, small, Type III lesions, as well as large subpial lesions, subdivided and quantified as two or more separate lesions. In addition to measuring the area of cortical GMLs, the area and relative extent of cortex identified as de/re-myelinated cortical grey matter was recorded per section, per case.

To produce illustrative "heat maps" that depict the burden of cortical GMLs in cases defined by lymphoidlike structure status (absence/presence), we first identified all cortical GMLs in section P1 per case and superimposed these lesions as "layer" images on a line-drawn representative whole coronal brain section (adapted from plate $34^{[22]}$ ) in GIMP. The final overlaid schema, comprising the "layer" masks of each case sampled at the P1 coronal level, revealed the absolute number of lesions by the relative depth of colour at that site.

\section{Determining leptomeningeal inflammation}

A measure of relative leptomeningeal immune cell infiltration per case was reported. Briefly, meningeal infiltrates were graded by assessing the extent of Nissl+ counter-stained cellular infiltrates of the intact cerebral leptomeninges, with the most notable infiltrate per case, rather than the average extent of infiltration, being reported. None to mild leptomeningeal inflammation was rated $0+(0-5$ cells per $100 \times$ microscopic field of view; equivalent to $440-\mu \mathrm{m}$ length of leptomeningeal tissue); diffuse and modest rated ++ (equivalent to an infiltrate of 5-50 loosely packed cells); or substantial infiltration rated +++ (based on $>50$ cells in a tightly packed infiltrate ${ }^{[18]}$ ). Bona fide leptomeningeal lymphoid-like structures characterised by the presence of an anti-CD35+ reticular network, proliferating B cells (Ki67 antigen + ) and immunoglobulin+ plasma cells ${ }^{[23]}$ were previously reported in a subset of these cases ${ }^{[18]}$ and the lymphoidlike structure status (presence or absence of detectable structures) is detailed in is detailed in the results section.

\section{Quantifying activated microglia/macrophages}

The density of CD68+ microglia/macrophages in cortical GML centres, normal appearing cortical grey matter or WML centre or normal appearing white matter was quantified from four $40 \times$ images (Zeiss 


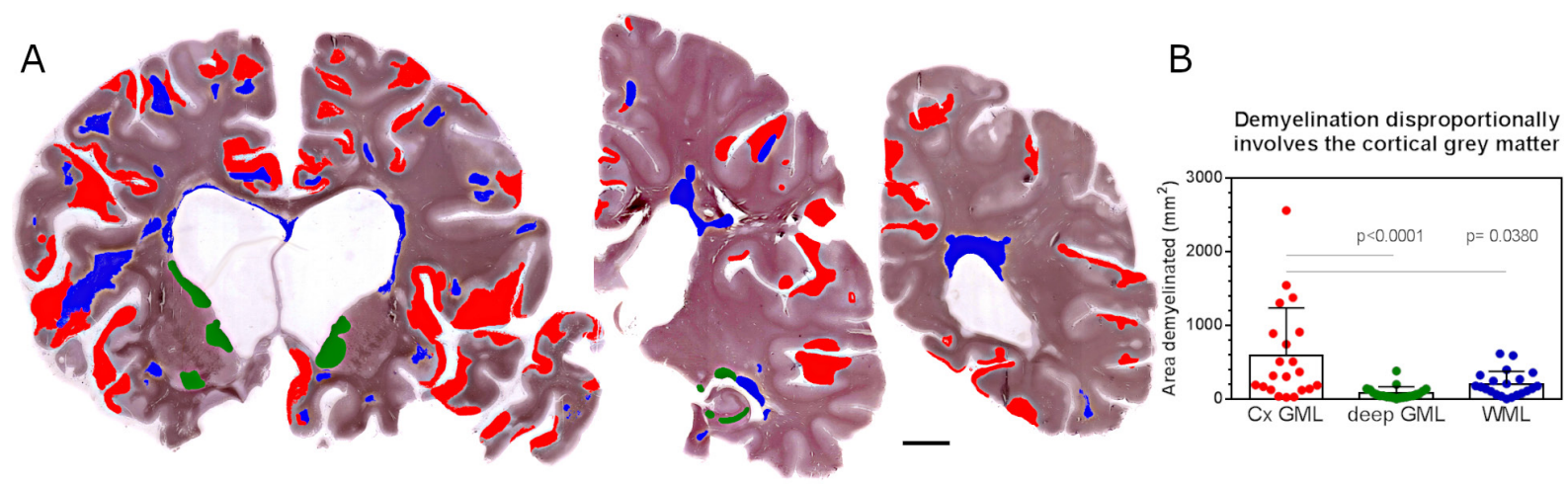

Figure 2. Demyelinating lesions of the cortical grey matter, deep grey matter and hippocampus and white matter. Anti-PLP immunostaining of sections through the rostral-caudal extent of MS212, at coronal levels A3, P1 and P3 (A). Note the widespread demyelination of the cortical grey matter (red colour mask). Quantification of the area of demyelinated tissues revealed the total area of demyelinated cortical grey matter to exceed that of the total measured area of deep grey matter and white matter (B). Data points represent the sum of all demyelinated lesions, per region of interest per case with mean and SD depicted. Groups compared by KruskalWallis and Dunn's multiple comparison post-test. Cx: cortical; GML: grey matter lesion; WML: white matter lesion. Scale bar $=1 \mathrm{~cm}$

AxioImager $\mathrm{Z}_{1}$ and Axiocam Hrc camera) per region of interest captured from cingulate, pre-central gyrus (including pre- and post-central superior gyrus), insula cortex in parietal lobe and temporal lobe (medial) of each available P1 coronal macrosection, per case. In the absence of a demyelinating lesion, only data from the normal appearing tissue were reported for that region, per case. All data were averaged for lesion or normal appearing status across the sampled regions and reported as density of CD68+ cells $/ \mathrm{mm}^{2}$, for comparison between groups.

\section{Statistical analysis}

Group means or medians were compared and plotted using GraphPad Prism 7. All data were assumed to be non-normally distributed given the small sample size ${ }^{[24]}$. The unpaired Kruskal-Wallis with Dunn's multiple comparison post-test was used for three-group comparisons [i.e., when comparing the average area $\left(\mathrm{mm}^{2}\right)$ of cortical and subcortical lesions per section, per case], whilst the Mann-Whitney $U$ test was used when comparing two groups (i.e., per cent hippocampus and deep GML in cortical High vs. Low MS). Fischer's exact test compared the actual $v s$. anticipated proportion of males/females and other clinical variables, such as seizures (yes/no) or lymphoid-like structure status (absent/present), between groups. Correlative analysis used Spearman's method and Spearman $r$ and $P$ values were reported. A two-tailed $P$ value of $>0.05$ was considered significant.

\section{RESULTS}

We conducted a study of whole coronal sections to better understand the burden of demyelination and inflammation in a cohort of 22 cases of secondary progressive MS (SPMS).

\section{Cortical grey matter is disproportionality demyelinated in progressive MS}

Cortical grey matter (GM), hippocampus and deep GM and WMLs were seen in all 22 cases analysed, with lesions noticeable across the coronal planes sampled [Figures 1 and $2 \mathrm{~A}$ ]. The area of demyelination, determined from the analysis of LFB and anti-PLP immuno-stained sections, varied considerably amongst the MS cohort [Figure 2B]. Quantification of the total area occupied by cortical GMLs, hippocampus and deep GML and WMLs revealed the total area of cortical GML (mean $589 \mathrm{~mm}^{2}$, range $25-2563 \mathrm{~mm}^{2}$ ) to be significantly greater than the total hippocampus and deep GML area (mean $81 \mathrm{~mm}^{2}$, range $0-382 \mathrm{~mm}^{2}$ ) and subcortical WML area (mean $203 \mathrm{~mm}^{2}$, range $2-617 \mathrm{~mm}^{2}$; Figure $2 \mathrm{~B}$ ). The relative area of cortical GML (per cent GML of total cortical GM) was almost twice that of the relative area of WML of total section WM per 


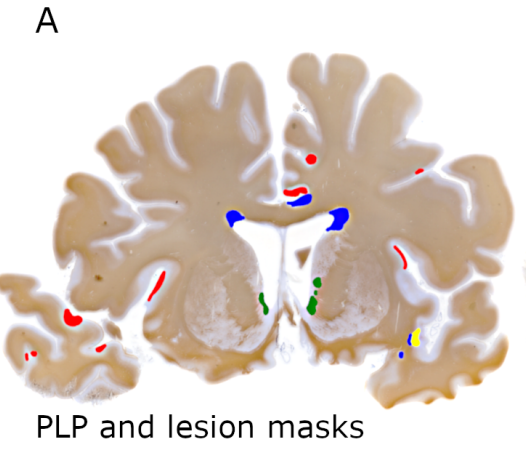

PLP and lesion masks

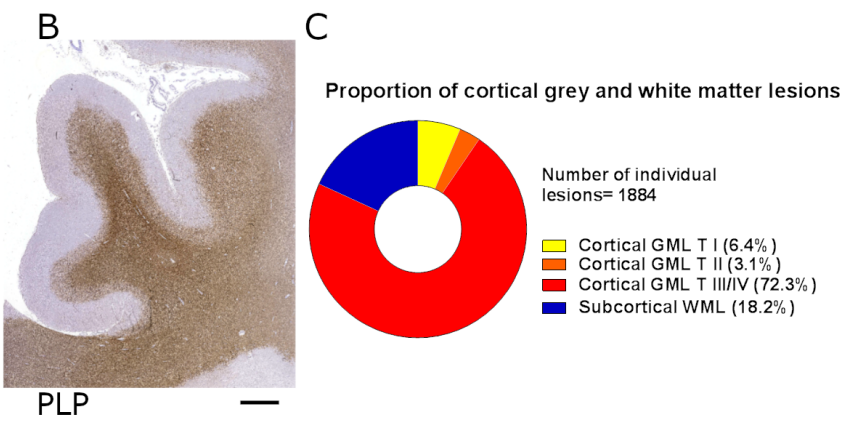

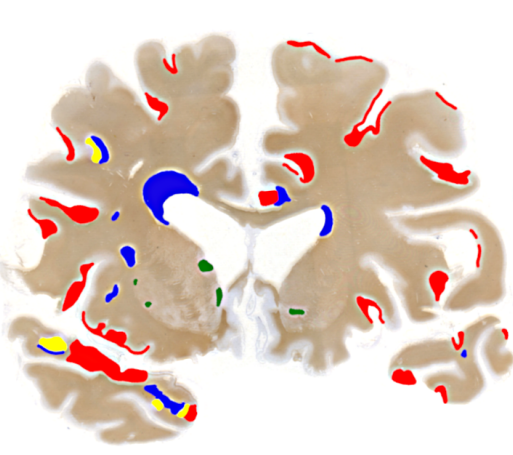
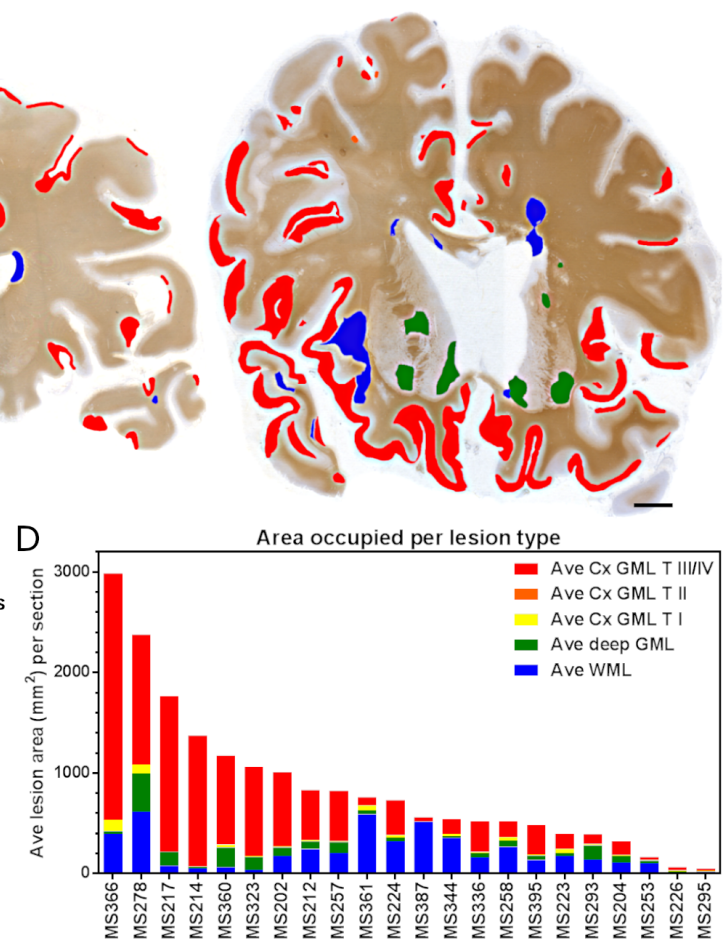

Figure 3. Subpial cortical grey matter lesions are the most frequent and occupy the greatest area. Anti-PLP immunostaining of section A3 of MS253, MS224 and MS257 revealed subpial (Type III/IV cortical lesions, red mask), intracortical (Type II cortical lesion, orange mask) and leukocortical (Type I cortical lesion, yellow mask) lesions. Lesions of the deep grey matter (green) and lesions of white matter (blue mask) are also shown (A). Subpial (Type III/IV) lesions were predominant and often spanned multiple gyri (B). Of 1884 separate lesions, $72.3 \%$ were subpial cortical grey matter lesions (C). By plotting the average area of each lesion type (calculated per section) and represented per case, we show the heterogeneity of lesion size across our cohort (D), and how subpial cortical lesions contribute a large component of the lesion burden, especially in those cases most affected (from MS366 to MS257, for example). Ave: average; Cx: cortical; GML: grey matter lesion; PLP: proteolipid protein immunohistochemistry; WML: white matter lesion. (A) Scale bar $=1 \mathrm{~cm}$; (B) Scale bar $=1 \mathrm{~mm}$

case $(17.9 \% \pm 2.6 \%$ compared with $8.9 \% \pm 1.3 \%$ for cortical GML and subcortical WML, respectively, $P=$ 0.023 , non-parametric Mann-Whitney $U$ test).

\section{Type III cortical grey matter lesions are the most frequent and can affect the entire cortical ribbon in a whole coronal section}

We noted cases with little and others with expansive areas of cortical GM demyelination in our progressive MS cohort [Figure 3A]. We counted the number of separate lesions of the neocortex and white matter and showed that the subpial cortical GMLs (Type III and IV lesions; Figure 3B) were more numerous than any other cortical grey matter lesion type or white matter lesion (Figure $3 \mathrm{C}, P<0.0001$ ). In total, 1884 lesions were identified, of which 1363 (72.3\%) were subpial (Type III or IV) cortical GMLs.

Subpial cortical GMLs accounted for the largest area of all lesion types measured. We plotted the measured area of each lesion type, with the bar height representing the total average area $\left(\mathrm{mm}^{2}\right)$ of demyelination per section, per case, to show the substantial contribution subpial GMLs made to the total lesion area [Figure 3D]. Arranging cases according to the total measured area of demyelination [from left (highest), to right (lowest)] highlighted that subpial GMLs overwhelmingly accounted for the different total lesion areas between cases with relatively high or low total demyelinated lesion area. In cases with relatively lower lesion area (cases MS361-MS295), the area of subpial GML as a per cent of total measured lesion area was far less than in cases with the higher total lesion area (34.5\%, range $8.0 \%-60.1 \% v s .74 .3 \%$, range $54.3 \%-94.9 \%$; $P$ $<0.0001$, non-parametric Mann-Whitney $U$ test). We compared the relative extent of demyelination in 
Table 3. Correlating cortical, white matter and hippocampus and deep grey matter lesions

\begin{tabular}{|c|c|c|c|c|}
\hline & \% Cx GML III/IV & \% Hippo and deep GML & $\% \mathbf{W M L}$ & \% Total Cx GML \\
\hline$\% \mathrm{C} \times \mathrm{GML} \mathrm{I} / \mathrm{II}$ & $\begin{array}{l}r=-0.005 \\
P=0.982\end{array}$ & $\begin{array}{l}r=0.224 \\
P=0.328\end{array}$ & $\begin{array}{l}r=0.771 \\
P<0.0001\end{array}$ & $\begin{array}{l}r=0.051 \\
P=0.827\end{array}$ \\
\hline$\%$ Cx GML III/IV & & $\begin{array}{l}r=0.458 \\
p=0.037\end{array}$ & $\begin{array}{l}r=0.092 \\
P=0.684\end{array}$ & $\begin{array}{l}r=0.988 \\
p<0.0001\end{array}$ \\
\hline $\begin{array}{l}\text { \% Hippo and deep } \\
\text { GML }\end{array}$ & & & $\begin{array}{l}r=0.169 \\
P=0.464\end{array}$ & $\begin{array}{l}r=0.495 \\
P=0.023\end{array}$ \\
\hline$\%$ WML & & & & $\begin{array}{l}r=0.135 \\
P=0.549\end{array}$ \\
\hline
\end{tabular}

Correlation analysis revealed the relative area of intracortical and leukocortical grey matter lesions (Type I/II cortical lesions) associated with the extent of white matter demyelination. Subpial cortical grey matter lesions (Type III/IV) associated with the extent of hippocampus and deep grey matter lesion area. Row and column headings refer to: \% CX GML I/II: relative area of intracortical/ leukocortical grey matter lesions; CX GML III/IV: relative area of subpial cortical grey matter lesions; \% Hippo and deep GML: relative area of grey matter lesions affecting hippocampus and deep grey matter; \% WML: relative area of demyelinated white matter; \% Total CX GML: relative area of demyelinated neocortex. Spearman analysis with $r$ and $P$ values shown. Significant associations are underlined. $C x$ : cortical; GML: grey matter lesion; WML: white matter lesion

cortical and subcortical tissues and showed that the relative area of total cortical GML was not statistically associated with the relative area of WML $(r=0.135, P=0.549$; Table 3). The area of subpial cortical GML was significantly associated with the extent of hippocampus and deep GML area (Spearman $r=0.458, P=$ 0.036), whilst leukocortical/intracortical GML area was significantly associated with WML area (Spearman $r=0.771, P<0.0001$; Table 3).

\section{Pathological correlates of a high subpial cortical grey matter lesion load}

To understand the clinical and pathological associations that might co-exist with subpial cortical GM demyelination, we subdivided the cohort based on relative extent of cortical GML area per section, per case [Table 4 and Figure 4]. Cases with 15\% or greater relative cortical GML of total cortical GM ( $n=9$ cases in total; Figure 4A, which represent cases MS366-MS257 in graph Figure 3D) were designated cortical GML High MS (mean cortical GML area 35.6\%; range 15.6\%-60.2\%), whilst the others ( $n=13$ cases, cortical GML less than $12 \%$ total cortical GM area) were designated as cortical GML Low MS (mean cortical GML area $5.5 \%$; range $0.9 \%-12.0 \%)$.

The relative area of hippocampus and deep GML was greater in cortical GML High MS [Figure 4B], whilst the relative area of WML was not different [Figure 4C]. Cortical GML High MS cases had a greater density of CD68+ microglia/macrophages in both GML centre and normal appearing GM regions, in comparison to cortical GML Low MS [Figure 4D and G], whilst the density of CD68+ microglia/macrophages was only increased in normal appearing WM of cortical GML High cases [Figure 4E and $\mathrm{H}$ ]. Inflammation of the forebrain leptomeninges is associated topographically with subpial cortical demyelination and cases with semi-organised lymphoid-like structures are characterised by a more extensive cortical GML load ${ }^{[13]}$. Our designated cortical GML High MS cohort presented with a substantially increased median rating of leptomeningeal immune cell infiltrates $(P=0.003$, Figure $4 \mathrm{~F})$, with seven of nine cases containing at least one bona fide lymphoid-like structure [Table 4]. Examples of leptomeningeal infiltrates rated as mild [+; Figure $4 \mathrm{I}$ and L], modest [++, Figure $4 \mathrm{~J}$ and $\mathrm{M}$ ] and substantial [+++, Figure $4 \mathrm{~K}$ and $\mathrm{N}$ ] are provided in Figure 4. Immune cell aggregates were associated both with areas of partially de/re-myelinated subpial cortical GM as well as areas of subpial GM demyelination [Figure 4].

Areas of cortical GM defined as partially de/re-myelinated GM ranged from < 1\% (0.9\%, MS223) to 13.5\% (MS257) of total cortical GM area per case but were not different between the cortical GML High and Low groups (area of partially de/re-myelinated GM $=8.01 \%$ vs. 6.13\%, for cortical High vs. Low GML cases, respectively, $P=0.387$ ). Rudimentary measures of gross brain pathology, such as brain weight at death and the measured area of grey and white matter per P1 section (as an indication of regional grey or white 
A

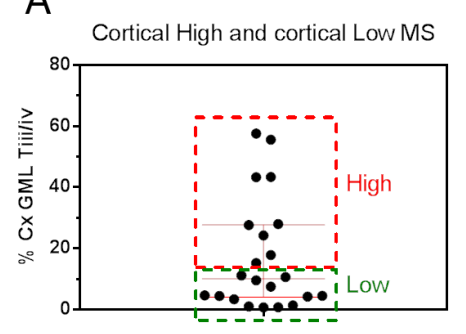

D Microglia/ macrophages in cortical

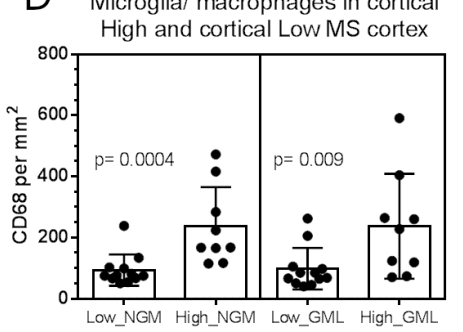

B

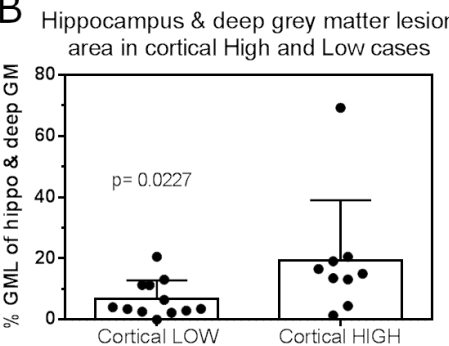

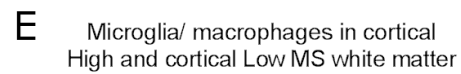

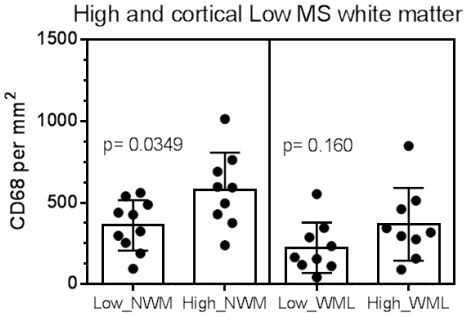

C White matter lesion area in cortical High and Low cases

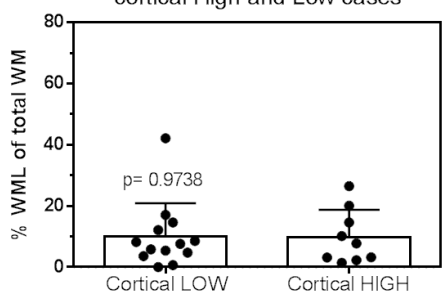

F Meningeal inflammation in cortical High and cortical Low MS

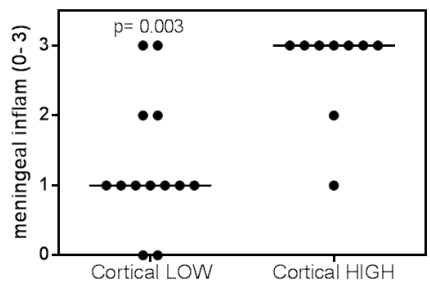

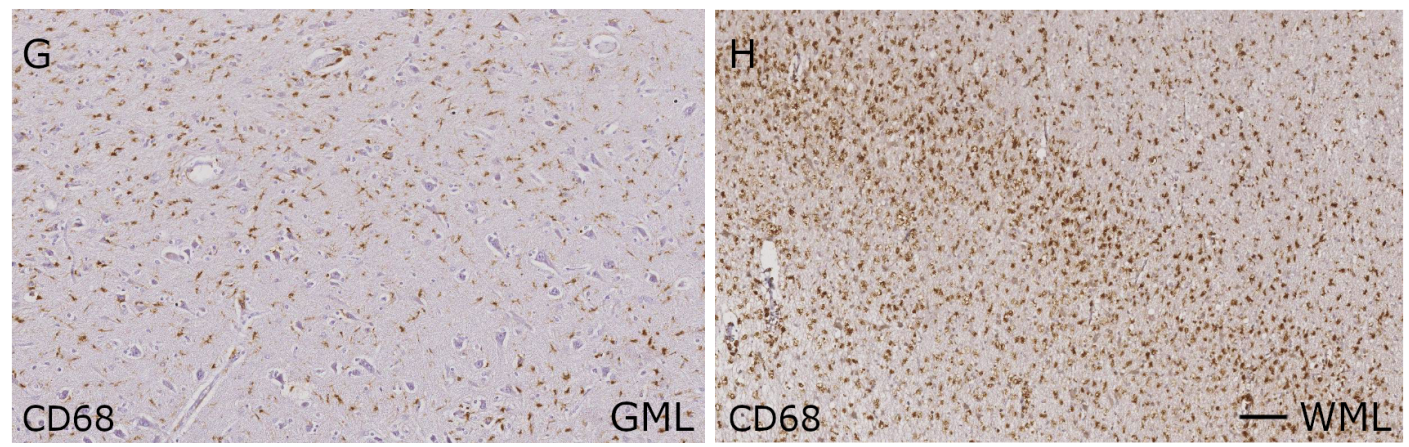
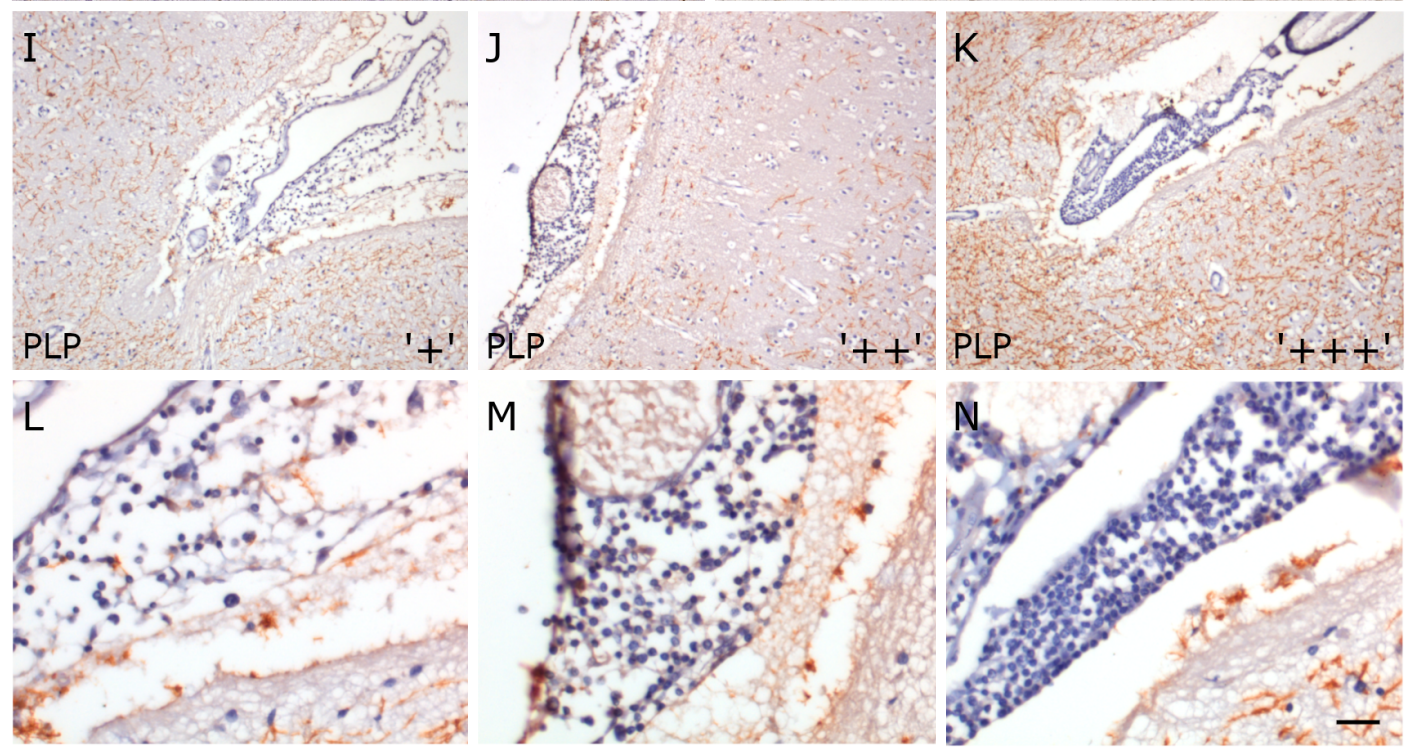

Figure 4. Defining a cohort of cortical High grey matter lesion MS. Nine progressive MS cases with the greatest relative area of subpial cortical demyelination were labelled as Cortical High GML MS and the remaining cases as Cortical Low GML MS (A). Cortical High GML MS had a greater area of demyelinated deep grey matter and hippocampus (B), but unchanged white matter lesion areas (C). The density of CD68+ microglia/macrophages was increased in both normal appearing GM (NGM) and GML of cortical High GML MS (D) but was only different in the underlying normal appearing WM (NWM) (E). Semi-quantitative evaluation of meningeal inflammation revealed cortical High GML MS to have increased inflammation of the leptomeninges (F). Examples of CD68+ microglia of a subpial GM lesion (G) and CD68+ microglia/macrophages in a white matter lesion (H). Examples of 1-3 rated inflammatory infiltrates (" + " to " $+++"$ ) of the forebrain meninges [I-N with (L-N) being higher power images of (I-K), respectively]. Please note that infiltrates $(I, L, K, N)$ are near partially de/re-myelinated cortex of cortical High GML Case MS257. Cx: cortical; GML: grey matter lesion; NGM: normal appearing grey matter; NWM: normal appearing white matter; PLP: proteolipid protein immunohistochemistry; WML: white matter lesion. (G-K) Scale bar $=100 \mu \mathrm{m} ;(\mathrm{L}-\mathrm{N})$ Scale bar $=25 \mu \mathrm{m}$ 
Table 4. Progressive MS cases defined by relative cortical grey matter lesion load

\begin{tabular}{|c|c|c|c|c|c|c|c|c|c|c|c|c|}
\hline Case & Sex & $2 y r R R$ & Onset & Prog & W'chair & Died & Duration & Pm delay & $\begin{array}{c}\text { Brain } \\
\text { weight (g) }\end{array}$ & $\begin{array}{l}\text { Seizures } \\
(Y / N)\end{array}$ & LLS & $\begin{array}{c}\text { Menin inflam } \\
(0-3)\end{array}$ \\
\hline \multicolumn{13}{|c|}{ Cortical GM High MS } \\
\hline MS202 & $\mathrm{F}$ & 2 & 35 & 46 & 47 & 58 & 23 & 39 & 1200 & $\mathrm{~N}$ & Y & 3 \\
\hline MS212 & $\mathrm{F}$ & 1 & 18 & 33 & 35 & 47 & 29 & 66 & 1314 & $\mathrm{~N}$ & Y & 3 \\
\hline MS214 & $\mathrm{F}$ & 2 & 20 & 31 & 38 & 51 & 31 & 20 & 1140 & $\mathrm{n} / \mathrm{d}$ & $\mathrm{N}$ & 2 \\
\hline MS217 & $\mathrm{F}$ & 1 & 42 & 49 & 50 & 57 & 15 & 29 & 1200 & $N$ & Y & 3 \\
\hline MS257 & $\mathrm{F}$ & 6 & 27 & 31 & 39 & 49 & 22 & 28 & 1168 & Y & Y & 3 \\
\hline MS278 & M & 1 & 9 & 23 & 23 & 30 & 21 & 60 & 1402 & Y & Y & 3 \\
\hline MS323 & $\mathrm{F}$ & 2 & 31 & 38 & 44 & 62 & 31 & 13 & 1207 & Y & $\mathrm{N}$ & 1 \\
\hline MS360 & M & 1 & 15 & 40 & 51 & 55 & 40 & 18 & 1063 & Y & Y & 3 \\
\hline MS366 & $\mathrm{F}$ & 3 & 42 & 45 & 53 & 61 & 19 & 14 & 1090 & $\mathrm{~N}$ & Y & 3 \\
\hline$n=9$ & $7 F: 2 M$ & 2 & 27 & 38 & 44 & 55 & 23 & 31.9 & 1198.2 & $4 Y: 4 N$ & $7 / 9$ & 3 \\
\hline \multicolumn{13}{|c|}{ Cortical GM Low MS } \\
\hline MS204 & M & 3 & 39 & 44 & 48 & 58 & 19 & 35 & 1180 & $\mathrm{~N}$ & $\mathrm{~N}$ & 1 \\
\hline MS223 & $\mathrm{F}$ & 4 & 43 & 43 & 45 & 45 & 2 & 72 & 1004 & Y & $\mathrm{N}$ & 1 \\
\hline MS224 & $\mathrm{F}$ & 1 & 26 & 35 & 36 & 59 & 33 & 27 & 1100 & $\mathrm{~N}$ & $\mathrm{~N}$ & 1 \\
\hline MS226 & $\mathrm{F}$ & 5 & 37 & 51 & 58 & 64 & 27 & 27 & 1300 & $\mathrm{~N}$ & $\mathrm{~N}$ & 0 \\
\hline MS253 & $\mathrm{F}$ & 3 & 21 & 23 & 33 & 37 & 16 & 24 & 1259 & $\mathrm{~N}$ & $\mathrm{~N}$ & 2 \\
\hline MS258 & M & 2 & 26 & 35 & 38 & 46 & 20 & 44 & 1460 & $\mathrm{~N}$ & $\mathrm{~N}$ & 1 \\
\hline MS293 & $\mathrm{F}$ & 2 & 35 & 42 & 43 & 53 & 18 & 44 & 1250 & Y & $Y$ & 3 \\
\hline MS295 & $\mathrm{F}$ & 5 & 56 & 60 & 62 & 71 & 15 & 42 & 1166 & $\mathrm{~N}$ & $\mathrm{~N}$ & 0 \\
\hline MS336 & $\mathrm{F}$ & 1 & 30 & 47 & 51 & 57 & 27 & 24 & 1226 & $\mathrm{~N}$ & Y & 3 \\
\hline MS344 & $\mathrm{F}$ & 6 & 42 & 43 & 47 & 57 & 15 & 14 & 1062 & $\mathrm{~N}$ & $\mathrm{~N}$ & 1 \\
\hline MS361 & $\mathrm{F}$ & 2 & 26 & 38 & 42 & 60 & 34 & 10 & 956 & Y & $\mathrm{N}$ & 1 \\
\hline MS387 & $\mathrm{F}$ & 6 & 32 & 35 & 36 & 43 & 11 & 13 & 1115 & $\mathrm{~N}$ & $N$ & 2 \\
\hline MS395 & $M$ & 1 & 37 & 45 & 52 & 63 & 26 & 4 & 958 & $\mathrm{~N}$ & $\mathrm{~N}$ & 1 \\
\hline$n=13$ & 10F:3M & 3 & 35 & 43 & 45 & 57 & 19 & 24.3 & 1161.3 & $3 Y: 10 N$ & $2 / 13$ & 1 \\
\hline
\end{tabular}

Associated clinical and pathological data of Cortical High and Cortical Low grey matter lesion MS. Sex (Female/Male), 2yr RR (number of relapses in first two years of clinical disease), Onset (retrospectively determined age at first MS symptoms, Prog (age at which disease became progressive), Died (age at death), Duration (time from first symptom onset to death), PM delay (post-mortem delay in hours), Brain weight (wet brain weight), Seizures (one or more seizures recorded in the clinical notes - Yes or No), LLS [positive (Yes) or negative (No)], Menin inflam (relative extent of meningeal inflammation). $\mathrm{n} / \mathrm{d}$, not determined, data not available. Text in bold represents median or mean (brain weight only) values for each group. LLS: lymphoid-like structure; GM: grey matter; MS: multiple sclerosis; W'chair: age at which they required a wheelchair

matter tissue atrophy; data not shown), did not reveal a difference between the cortical GML High and Low MS cases [Table 4].

\section{Clinical correlations of a high subpial cortical grey matter lesion load}

There was no difference in the proportion of males to females or of any reported clinical measure (such as the number of relapses in the first two years, the report of seizures or age of death) between cortical GML High and Low groups [Table 4]. There was no difference between groups with regards age of disease onset $(P$ $=0.11)$, confirmed age at onset of progressive phase $(P=0.27)$ or disease duration $(P=0.15)$. Post-mortem delay did not differ between the groups $(P=0.75$; Table 4$)$.

\section{Leptomeningeal inflammation, lymphoid-like structures and cortical demyelination}

We identified four separate "+++" rated foci of substantial leptomeningeal infiltrates in a single P1 section of Case MS217, which displayed massive cortical, and exclusively subpial, GM demyelination (59.6\% of cortical GM defined as GML; Figure 5A and B). Progressive MS cases with moderate-to-high leptomeningeal inflammation (rated $++/+++$ or $+++/+++$ for immune cell infiltrates) had an increased relative cortical GML area compared with those cases with little-to-mild leptomeningeal infiltrates $7.8 \%$ (range $0.8 \%-27.7 \%$ ) vs. 26.2\% (range 0.8\%-60.2\%), $P=0.012$, Mann-Whitney $U$ test). The relative area of hippocampus and deep GML area $(7.4 \%$, range $0 \%-19.1 \%$ vs. $16.4 \%$ range $1.4 \%-69.2 \%, P=0.159)$ and WML 

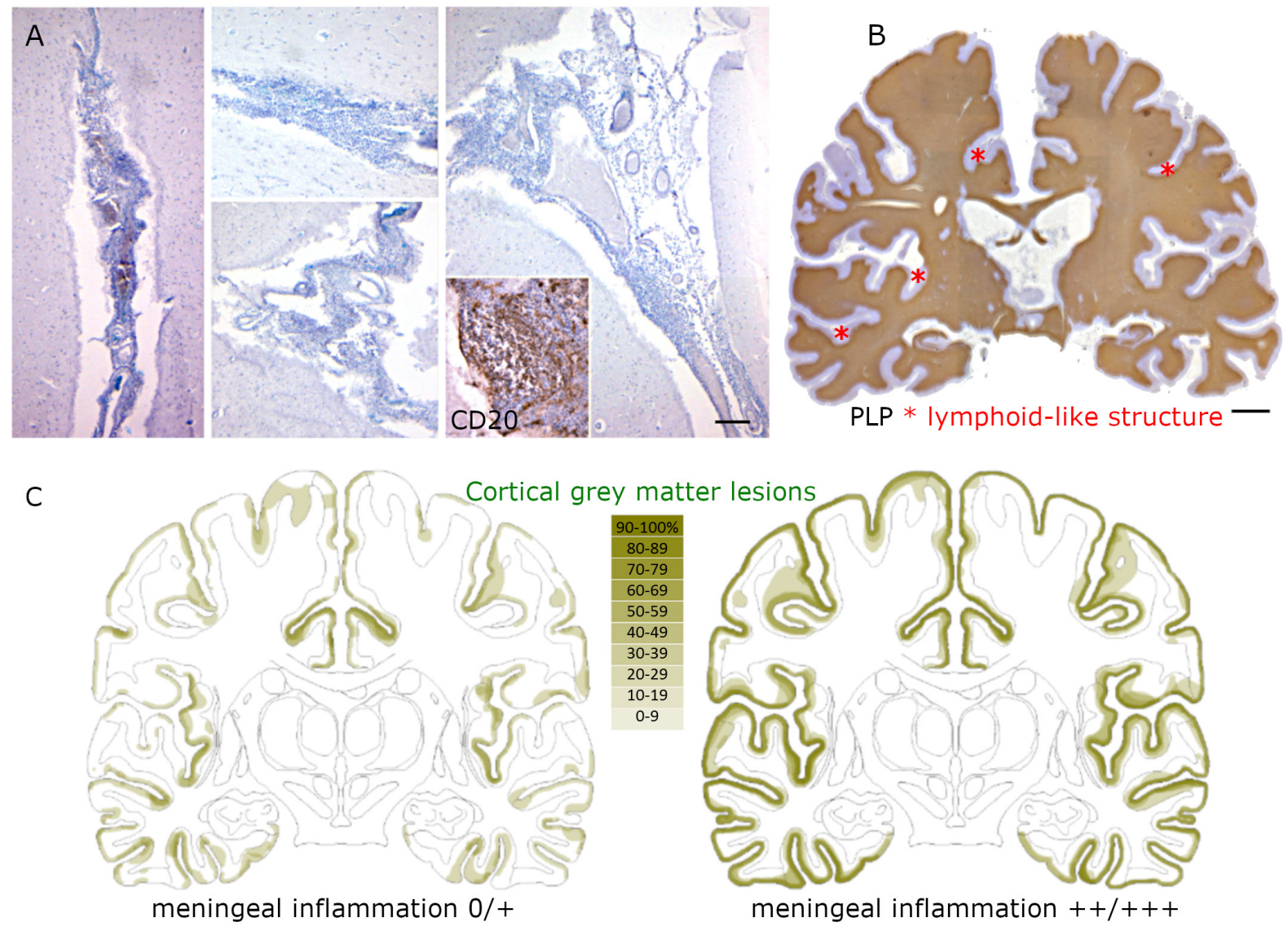

Figure 5. Meningeal inflammation and subpial cortical demyelination. Example infiltrates of the leptomeninges overlying the neocortex in a single coronal macrosection. Aggregates visualised by LFB/haematoxylin histology were rich in CD20+ B cells ( $A$ ). The location of the infiltrates in (A) are marked as asterisks $\left(^{\star}\right)$ on the serial anti-PLP stained section (B) (Case MS217). By grouping the cases based on their relative extent of leptomeningeal inflammation $(0 /+v s .++/+++)$, we constructed schema depicting all neocortical grey matter lesions noted per P1 section, per case $(n=18)$. Depth of colour (see heatmap representing percent lesion occurrence with $100 \%$ indicating tissue was demyelinated in every P1 section in that group) illustrates how frequently an area of neocortex was demyelinated in meningeal inflammation rated $0 /+$ and meningeal inflammation rated $++/+++$ MS (C). PLP: proteolipid protein. (A) Scale bar $=200$ $\mu \mathrm{m}$; (B) Scale bar $=1 \mathrm{~cm}$

lesion area (10.1\%, range $0.6 \%-42.1 \%$ vs. $10.0 \%$, range $2.3 \%-26.5 \%, P=0.981$ ) was unchanged between leptomeningeal rated moderate-high cases $v s$. those rated with little-to-mild infiltrates, respectively. The relationship between the extent of leptomeningeal inflammation and cortical GML area is presented schematically as a heat map of all cortical GMLs observed (in olive green colour) in section P1 of cases with mild (rated 0 to,$+ n=9$ ) vs. modest/substantial infiltrates (++ to,$+++ n=9$; Figure 5 C). Please note that four cases did not have P1 sections available for analysis [Table 1].

\section{DISCUSSION}

Our quantitative histological analysis of whole coronal macrosections demonstrates cortical grey matter demyelination can be the preeminent pathology in some cases of progressive MS and can be used to identify a post-mortem cohort with a more severe pathological disease. Subpial cortical grey matter lesions associated with the extent of demyelinated deep grey matter and with infiltrates of the leptomeninges but importantly did not associate with white matter lesion load. Our study reinforces the concept of a CSFderived (outside-in) driver of tissue pathology in SPMS. 


\section{Substantial subpial cortical grey matter demyelination defines a subset of progressive MS cases}

Quantitative image analysis of whole coronal macrosections revealed the proportion of demyelinated cortical grey matter to be almost twice that of the proportion of demyelinated white matter. In those cases, with the greatest cortical pathology, labelled as cortical GML High MS, there was a near four-fold increase in relative area of cortical GML compared to WML. These data are in accordance with the work of Carassiti et al. ${ }^{[25]}$ and are supportive of an MS post-mortem cohort characterised by a dominant neocortical demyelinating pathology ${ }^{[26]}$. Amongst our samples, we observed instances of hugely divergent grey over white matter pathology (for example, Case MS214 WML accounted for 3.2\% of total WM, whilst cortical GML area was $43.4 \%$ of total cortex; Case MS217 WML area was 3.1\% and cortical GML was 59.6\%). Cortical GML pathology was overwhelmingly subpial in location, with an average of $90.9 \%$ of total cortical GML being characterised as subpial (Type III/IV) in distribution. Such extensive and disproportionate demyelination exceeds that previously reported by ourselves and others and highlights the benefit of working with whole coronal macrosection preparations ${ }^{[2,12,18]}$ to better understand MS pathology.

We have previously noted associations between lymphoid-like structure status and clinical disease measures, such as age of onset, age at progression and age at death ${ }^{[13,18]}$. Our cortical GML High and Low MS cases had a similar age of onset to that seen in our earlier studies (median age at onset of 27 and 35 years, respectively) but this did not represent a significant difference in our relatively small study. No other clinical-pathological correlation was noted, which in part reflects the complex and highly variable pathology of this disease, whereby synaptic, neuritic and neuronal loss, alongside demyelination and gliosis, variable impact on disease outcome.

\section{Demyelinating pathology at subpial and subependymal territories}

The correlation between the extent of demyelination of the hippocampus and deep grey (including the caudate, putamen, pallidum and thalamus) with that of subpial cortical GML suggests, at least in part, a shared pathological mechanism of lesion formation and/or expansion between these regions lining superficial surfaces of the pia and ventricles. Subpial demyelinating lesions are a pathological hallmark of $\mathrm{MS}^{[27]}$ and clinical imaging shows that the thalamus of children with MS, a structure which is severely affected in progressive $\mathrm{MS}^{[28]}$, displays a specific imaging abnormality as a surface-in gradient pattern from the ventricular margin ${ }^{[29]}$. Adult MS patients also display a gradient of magnetisation transfer ratio signal change from the superficial surfaces of the brain: a gradient of signal change, declining with distance across the white matter ${ }^{[30,31]}$ when measured from the ventricular surface, and across the neocortex from the pial surface, which are most altered in progressive $\mathrm{MS}^{[32,33]}$. A magnetic resonance imaging (MRI) signature of gadolinium leptomeningeal enhancement, which may partly reflect inflammation of meninges ${ }^{[34]}$, relates to the number and volume of cortical and thalamic lesions ${ }^{[35]}$. These imaging studies support the concepts of meningeal inflammation and outside-in CSF factor(s) in lesion genesis. Numerous post-mortem studies have demonstrated a topographical association between the extent of immune cell infiltration of the leptomeninges with cortical GM demyelination ${ }^{[13,23,36-40]}$, which is also true for subpial tissue of the cerebellar cortex and spinal cord ${ }^{[41-44]}$. This subpial demyelinating pathology of the neocortex is associated with microglia activation, a gradient of tissue injury and disruption of the pial glial limiting membrane ${ }^{[16,45]}$. Therefore, the pattern of neocortical lesion location and deep GM pathology is suggestive, at least in part, of an effect of soluble cytotoxic mediators from the overlying CSF-filled spaces that contributes to the underlying disease process.

\section{Soluble mediators of subpial lesions}

Recently, we have demonstrated the overexpression of RNA transcripts associated with pleiotropic chemokines and cytokines in the isolated meningeal tissue from cases characterised by extensive leptomeningeal inflammation and subpial demyelination ${ }^{[46,4]}$. The profile of elevated mediators, many 
of which are associated with processes of lymphoid neogenesis, were mirrored by the finding of elevated protein levels of many of the same factors in the matched post-mortem CSF, which were also differentially expressed in an independent cohort of newly diagnosed MS patients with a clinical and radiological signature of substantial cortical pathology ${ }^{[47]}$. TNF and IFN $\gamma$ are amongst those differentially expressed in patients with a cortical phenotype and these cytokines mediate a rapid pattern of cortical demyelination and microglia/macrophage activation when injected into the subarachnoid space of animals with subclinical autoimmune encephalitis ${ }^{[46]}$. These findings imply that lymphoid follicle-like structures, and infiltrates outside of these semi-organised structures, are a source of damaging factors that drive subpial pathology. Sampling CSF may reveal disease-relevant biomarkers of activity to aid therapeutic decision making $^{[48]}$.

Subpial cortical GMLs are associated with the loss of neurons of the superficial cortical layers, an elevated number of complement activated neurons and glia, together with substantial neuritic and synaptic $\operatorname{loss}^{[13,16,45,49-53]}$. The most striking pathological changes are found in the superficial cortical laminae, with a gradient of lessening neuronal damage with distance from the pia ${ }^{[16]}$. Neurons and glia of the MS cortex are exposed to elevated excitotoxins and reactive free radicals ${ }^{[54,55]}$, are energy depleted and display mitochondrial pathology, which may contribute to further neurodegeneration ${ }^{[56-58]}$. There is an imbalance between TNF receptor $1 / 2$ anti-apoptotic pathways $v s$. pro-death signals in the progressive MS cortex and oligodendrocytes are vulnerable to degenerate by a TNF receptor type 1/Receptor-interacting protein kinase type 1 necroptotic cascade ${ }^{[59,60]}$. Immunoglobulins derived from meningeal plasma cells are enriched in the MS neocortex, and products of central CD20+ B effector cells are directly toxic to cultured neurons and oligodendrocytes ${ }^{[61-63]}$. Alongside myriad immune mediators, MS CSF is enriched in bio-active lipids, whose levels associate with disease severity ${ }^{[64-66]}$. Lipid sterols, including key products of cholesterol metabolism and ceramide, can be directly neurotoxic. For example, C16:0 and C24:0 ceramides are enriched in MS patient CSF and can mediate neuroaxonal pathology and mitochondrial dysfunction ${ }^{[65]}$, whilst simvastatin, a cholesterol-reducing therapy that enters the CNS, is associated with a slowing of brain atrophy in $\mathrm{SPMS}^{[67]}$. We currently have identified neither the combination of damaging factors that are causative of injury nor the relationship between these mediators with clinical progression on an individual level.

Lesions of the deeper cortical laminae (leukocortical or Type I cortical GMLs) are centred on cortical veins, typically contain greater numbers of activated microglia/macrophages than subpial lesions ${ }^{[53]}$, and their relative area correlated with the extent of white matter lesion area in the same case. The statistical association between cortical and subcortical lesions, both characterised by an inflamed central vein, suggests they both share similar a mechanism of formation, which is in part different to lesions of the superficial grey matter structures. MRI is adept at resolving leukocortical GMLs, whilst even ultra-high field MRI detects only a fraction of all subpial lesions ${ }^{[68]}$. Our finding of an association between the relative area of leukocortical GML and WML area may explain the correlation between white matter and cortical grey matter lesions reported by MRI.

\section{Methodological considerations and study limitations}

The analysis of whole coronal macrosections allows the study of cortical lesions in continuity, improves the accuracy of their interpretation and the relationship with other pathological features, such as meningeal inflammation or lesions in different anatomical sites ${ }^{[19,69,70]}$. The handling of such large tissue samples is not trivial and this restricted the $n$ number for our work, which may have meant our clinical-pathological comparisons were statistically underpowered [for instance, to observe a significant difference in age of onset between our GML High and Low MS cases, post-hoc power calculations $(\beta=0.8)$ predicted a cohort of at least 40 cases would be required $(\alpha=0.05)$ ]. Whole brain immersion fixation often led to some deformation of tissues, such that it was not always possible to align anatomical structures of 
interest, meaning that we could not, for example, report lesion measures for separate deep GM structures. Nevertheless, the use of whole coronal sections reduces observer variation between individual cases, increases the accuracy of observations, and ensures comparisons between different forebrain areas, which are invaluable for the study of this heterogeneous disease.

In conclusion, our quantitative histological analysis revealed global grey matter demyelination and meningeal inflammation to be substantial in a subset of progressive MS brains. Cases defined by a substantial cortical GML load displayed greater microglia/macrophage activation, larger areas of deep grey matter pathology but little change in white matter lesion area, which highlights the partially separate pathogenetic mechanisms of lesion evolution (or susceptibility to damage) in these compartments. The distribution of subcortical and deep GML in MS is consistent with the presence of soluble proinflammatory factors in the subarachnoid space and ventricular CSF and furthers the need to identify companion biomarkers of cortical pathology to aid patient monitoring and therapeutic choice.

\section{DECLARATIONS}

\section{Authors' contributions}

Made substantial contributions to conception and design of the study: Griffiths L, Reynolds R, Howell OW Performed data analysis, interpretation and contributed to writing the manuscript and approved the final submitted document: Griffiths L, Reynolds R, Evans R, Bevan RJ, Rees MI, Gveric D, Neal JW, Howell OW

\section{Availability of data and materials}

Data generated during the current study is available on reasonable request.

\section{Financial support and sponsorship}

The UK MS Society Tissue Bank (www.ukmstissuebank.imperial.ac.uk) supplied the post-mortem samples used in this study and is supported by the Multiple Sclerosis Society of Great Britain and Northern Ireland. The authors would like to thank members of the UK MS Tissue Bank for their invaluable assistance. This work was supported by the St. Davids Medical Foundation, the British Neuropathological Society and the Medical Research Council (G0700356). Reynolds R has received speaking honoraria from Roche, Novartis and ECTRIMS and grant funding from MedImmune plc. Howell OW has received travel reimbursement or speaking honoria (paid to Swansea University) from Roche, the Neurology Academy and ECTRIMS. All other authors have no relevant disclosures.

\section{Conflicts of interest}

All authors declared that there are no conflicts of interest.

\section{Ethical approval and consent to participate}

The use of human post mortem tissue is covered by UK Research Ethics committee approval (study approval number 08/MRE09/31+5).

\section{Consent for publication}

Not applicable.

\section{Copyright}

(c) The Author(s) 2020.

\section{REFERENCES}

1. Reynolds R, Roncaroli F, Nicholas R, Radotra B, Gveric D, et al. The neuropathological basis of clinical progression in multiple sclerosis. Acta Neuropathol 2011;122:155-70. 
2. Kutzelnigg A, Lucchinetti CF, Stadelmann C, Brück W, Rauschka H, et al. Cortical demyelination and diffuse white matter injury in multiple sclerosis. Brain 2005;128:2705-12.

3. Lucchinetti CF, Popescu BFG, Bunyan RF, Moll NM, Roemer SF, et al. Inflammatory cortical demyelination in early multiple sclerosis. N Engl J Med 2011;365:2188-97.

4. Bevan RJ, Evans R, Griffiths L, Watkins LM, Rees MI, et al. Meningeal inflammation and cortical demyelination in acute multiple sclerosis. Ann Neurol 2018;84:829-42.

5. Kutzelnigg A, Faber-Rod JC, Bauer J, Lucchinetti CF, Sorensen PS, et al. Widespread demyelination in the cerebellar cortex in multiple sclerosis. Brain Pathol 2007;17:38-44.

6. Calabrese M, Magliozzi R, Ciccarelli O, Geurts JJ, Reynolds R, et al. Exploring the origins of grey matter damage in multiple sclerosis. Nat Rev Neurosci 2015;16:147-58.

7. Calabrese M, Romualdi C, Poretto V, Favaretto A, Morra A, et al. The changing clinical course of multiple sclerosis: a matter of gray matter. Ann Neurol 2013;74:76-83.

8. Dalton CM, Chard DT, Davies GR, Miszkiel KA, Altmann DR, et al. Early development of multiple sclerosis is associated with progressive grey matter atrophy in patients presenting with clinically isolated syndromes. Brain 2004;127:1101-7.

9. Calabrese M, Rinaldi F, Mattisi I, Bernardi V, Favaretto A, et al. The predictive value of gray matter atrophy in clinically isolated syndromes. Neurology 2011;77:257-63.

10. Fisniku LK, Chard DT, Jackson JS, Anderson VM, Altmann DR, et al. Gray matter atrophy is related to long-term disability in multiple sclerosis. Ann Neurol 2008;64:247-54.

11. Scalfari A, Romualdi C, Nicholas RS, Mattoscio M, Magliozzi R, et al. The cortical damage, early relapses, and onset of the progressive phase in multiple sclerosis. Neurology 2018;90:e2107-18.

12. Vercellino M, Plano F, Votta B, Mutani R, Giordana MT, et al. Grey matter pathology in multiple sclerosis. J Neuropathol Exp Neurol 2005;64:1101-7.

13. Magliozzi R, Howell O, Vora A, Serafini B, Nicholas R, et al. Meningeal B-cell follicles in secondary progressive multiple sclerosis associate with early onset of disease and severe cortical pathology. Brain 2007;130:1089-104.

14. Pitzalis C, Jones GW, Bombardieri M, Jones SA. Ectopic lymphoid-like structures in infection, cancer and autoimmunity. Nat Rev Immunol 2014;14:447-62.

15. Bø L, Vedeler C, Nyland H, rapp BD, Mørk SJ. Intracortical multiple sclerosis lesions are not associated with increased lymphocyte infiltration. Mult Scler 2003;9:323-31.

16. Magliozzi R, Howell OW, Reeves C, Roncaroli F, Nicholas R, et al. A Gradient of neuronal loss and meningeal inflammation in multiple sclerosis. Ann Neurol 2010;68:477-93.

17. Lassmann H. Pathogenic mechanisms associated with different clinical courses of multiple sclerosis. Front Immunol 2019;10:1-14.

18. Howell OW, Reeves CA, Nicholas R, Carassiti D, Radotra B, et al. Meningeal inflammation is widespread and linked to cortical pathology in multiple sclerosis. Brain 2011;134:2755-71.

19. Haider L, Zrzavy T, Hametner S, Höftberger R, Bagnato F, et al. The topograpy of demyelination and neurodegeneration in the multiple sclerosis brain. Brain 2016;139:807-15.

20. Bø L, Vedeler C a, Nyland HI, Trapp BD, Mørk SJ. Subpial demyelination in the cerebral cortex of multiple sclerosis patients. J Neuropathol Exp Neurol 2003;62:723-32.

21. Lassmann H, Raine CS, Antel J, Prineas JW. Immunopathology of multiple sclerosis: report on an international meeting held at the Institute of Neurology of the University of Vienna. J Neuroimmunol 1998;86:213-7.

22. Mai JK, George Paxinos TV. Atlas of the Human Brain. 3rd edition. Academic Press; 2007.

23. Serafini B, Rosicarelli B, Magliozzi R, Stigliano E, Aloisi F. Detection of ectopic B-cell follicles with germinal centers in the meninges of patients with secondary progressive multiple sclerosis. Brain Pathol 2004;14:164-74.

24. Motulsky HJ. 'When to choose a nonparametric test'. GraphPad Stat. Guid. Available from: https://www.graphpad.com/guides/prism/8/ statistics/index.htm [Last accessed on 2 Mar 2020]

25. Carassiti D, Altmann DR, Petrova N, Pakkenberg B, Scaravilli F, et al. Neuronal loss, demyelination and volume change in the multiple sclerosis neocortex. Neuropathol Appl Neurobiol 2018;44:377-90.

26. Trapp BD, Vignos M, Dudman J, Chang A, Fisher E, et al. Cortical neuronal densities and cerebral white matter demyelination in multiple sclerosis: a retrospective study. Lancet Neurol 2018;17:870-84.

27. Moll NM, Rietsch AM, Ransohoff AJ, Cossoy MB, Huang D, et al. Cortical demyelination in PML and MS: similarities and differences. Neurology 2008;70:336-43.

28. Cifelli A, Arridge M, Jezzard P, Esiri MM, Palace J, et al. Thalamic neurodegeneration in multiple sclerosis. Ann Neurol 2002;52:650-3.

29. Fadda G, Brown RA, Magliozzi R, Aubert-Broche B, O’Mahony J, et al. A surface-in gradient of thalamic damage evolves in pediatric multiple sclerosis. Ann Neurol 2019;85:340-51.

30. Liu Z, Pardini M, Yaldizli O, Sethi V, Muhlert N, et al. Magnetization transfer ratio measures in normal-appearing white matter show periventricular gradient abnormalities in multiple sclerosis. Brain 2015;138:1239-46.

31. Kearney H, Yiannakas MC, Samson RS, Wheeler-Kingshott CA, Ciccarelli O, et al. Investigation of magnetization transfer ratio-derived pial and subpial abnormalities in the multiple sclerosis spinal cord. Brain 2014;137:2456-68.

32. Samson RS, Cardoso MJ, Muhlert N, Sethi V, Wheeler-Kingshott CA, et al. Investigation of outer cortical magnetisation transfer ratio abnormalities in multiple sclerosis clinical subgroups. Mult Scler 2014;20:1322-30.

33. Mainero C, Louapre C, Govindarajan ST, Giannì C, Nielsen AS, et al. A gradient in cortical pathology in multiple sclerosis by in vivo 
quantitative 7 T imaging. Brain 2015;138:932-45.

34. Absinta M, Vuolo L, Rao A, Nair G, Sati P, et al. Gadolinium-based MRI characterization of leptomeningeal inflammation in multiple sclerosis. Neurology 2015;85:18-28.

35. Zurawski J, Tauhid S, Chu R, Khalid F, Healy BC, et al. 7T MRI cerebral leptomeningeal enhancement is common in relapsing-remitting multiple sclerosis and is associated with cortical and thalamic lesions. Mult Scler 2020;26:177-87.

36. Pirko I, Lucchinetti CF, Sriram S, Bakshi R. Gray matter involvement in multiple sclerosis. Neurology 2007;68:634-42.

37. Lucchinetti CF, Popescu BFG, Bunyan RF, Moll NM, Roemer SF, et al. Inflammatory cortical demyelination in early multiple sclerosis. N Engl J Med 2011;365:2188-97.

38. Guseo A, Jellinger K. The significance of perivascular infiltrations in multiple sclerosis. J Neurol 1975;211:51-60.

39. Adams CW. Pathology of multiple sclerosis: progression of the lesion. Br Med Bull 1977;33:15-20.

40. Dawson JW. XVIII.-the histology of disseminated sclerosis. Trans R Soc Edinburgh 1916;50:517-740.

41. Howell OW, Schulz-Trieglaff EK, Carassiti D, Gentleman SM, Nicholas R, et al. Extensive grey matter pathology in the cerebellum in multiple sclerosis is linked to inflammation in the subarachnoid space. Neuropathol Appl Neurobiol 2015;41:798-813.

42. DeLuca GC, Alterman R, Martin JL, Mittal A, Blundell S, et al. Casting light on multiple sclerosis heterogeneity: the role of HLA-DRB1 on spinal cord pathology. Brain 2013;136:1025-34.

43. Androdias G, Reynolds R, Chanal M, Ritleng C, Confavreux C, et al. Meningeal T cells associate with diffuse axonal loss in multiple sclerosis spinal cords. Ann Neurol 2010;68:465-76.

44. Brown DA, Sawchenko PE. Time course and distribution of inflammatory and neurodegenerative events suggest structural bases for the pathogenesis of experimental autoimmune encephalomyelitis. J Comp Neurol 2007;502:236-60.

45. Choi SR, Howell OW, Carassiti D, Magliozzi R, Gveric D, et al. Meningeal inflammation plays a role in the pathology of primary progressive multiple sclerosis. Brain 2012;135:2925-37.

46. Gardner C, Magliozzi R, Durrenberger PF, Howell OW, Rundle J, et al. Cortical grey matter demyelination can be induced by elevated pro-inflammatory cytokines in the subarachnoid space of MOG-immunized rats. Brain 2013;136:3596-608.

47. Magliozzi R, Howell OW, Nicholas R, Cruciani C, Castellaro M, et al. Inflammatory intrathecal profiles and cortical damage in multiple sclerosis. Ann Neurol 2018;83:739-55.

48. Magliozzi R, Hametner S, Facchiano F, Marastoni D, Rossi S, et al. Iron homeostasis, complement, and coagulation cascade as CSF signature of cortical lesions in early multiple sclerosis. Ann Clin Transl Neurol 2019;6:2150-63.

49. Schirmer L, Velmeshev D, Holmqvist S, Kaufmann M, Werneburg S, et al. Neuronal vulnerability and multilineage diversity in multiple sclerosis. Nature 2019;573:75-82.

50. Watkins LM, Neal JW, Loveless S, Michailidou I, Ramaglia V, et al. Complement is activated in progressive multiple sclerosis cortical grey matter lesions. J Neuroinflammation 2016;13:161.

51. Klaver R, Popescu V, Voorn P, Galis-de Graaf Y, van der Valk P, et al. Neuronal and axonal loss in normal-appearing gray matter and subpial lesions in multiple sclerosis. J Neuropathol Exp Neurol 2015;74:453-8.

52. Jürgens T, Jafari M, Kreutzfeldt M, Bahn E, Brück W, et al. Reconstruction of single cortical projection neurons reveals primary spine loss in multiple sclerosis. Brain 2016;139:39-46.

53. Peterson JW, Bö L, Mörk S, Chang A, Trapp BD. Transected neurites, apoptotic neurons, and reduced inflammation in cortical multiple sclerosis lesions. Ann Neurol 2001;50:389-400.

54. Fischer MT, Wimmer I, Höftberger R, Gerlach S, Haider L, et al. Disease-specific molecular events in cortical multiple sclerosis lesions. Brain 2013;136:1799-815.

55. Haider L, Fischer MT, Frischer JM, Bauer J, Höftberger R, et al. Oxidative damage in multiple sclerosis lesions. Brain 2011;134:1914-24.

56. Witte ME, Nijland PG, Drexhage JAR, Gerritsen W, Geerts D, et al. Reduced expression of PGC-1 $\alpha$ partly underlies mitochondrial changes and correlates with neuronal loss in multiple sclerosis cortex. Acta Neuropathol 2013;125:231-43.

57. Lassmann H, van Horssen J, Mahad D. Progressive multiple sclerosis: pathology and pathogenesis. Nat Rev Neurol 2012;8:647-56.

58. Campbell G., Ziabreva I, Reeve A, Krishnan KJ, Reynolds R, et al. Mitochondrial DNA deletions and neurodegeneration in multiple sclerosis. Ann Neurol 2011;69:481-92.

59. Ofengeim D, Ito Y, Trapp B, Zhang Y, Shan B, et al. Activation of necroptosis in multiple sclerosis. Cell Rep 2015;10:1836-49.

60. Magliozzi R, Howell OW, Durrenberger P, Aricò E, James R, et al. Meningeal inflammation changes the balance of TNF signalling in cortical grey matter in multiple sclerosis. J Neuroinflammation 2019;16:259.

61. Lisak RP, Benjamins JA, Nedelkoska L, Barger JL, Ragheb S, et al. Secretory products of multiple sclerosis B cells are cytotoxic to oligodendroglia in vitro. J Neuroimmunol 2012;246:85-95.

62. Lisak RP, Nedelkoska L, Benjamins JA, Schalk D, Bealmear B, et al. B cells from patients with multiple sclerosis induce cell death via apoptosis in neurons in vitro. J Neuroimmunol 2017;309:88-99.

63. Torkildsen Ø, Stansberg C, Angelskår SM, Kooi EJ, Geurts JJ, et al. Upregulation of immunoglobulin-related genes in cortical sections from multiple sclerosis patients. Brain Pathol 2010;20:720-9.

64. Crick PJ, Griffiths WJ, Zhang J, Beibel M, Abdel-Khalik J, et al. Reduced plasma levels of 25-hydroxycholesterol and increased cerebrospinal fluid levels of bile acid precursors in multiple sclerosis patients. Mol Neurobiol 2017;54:8009-20.

65. Vidaurre OG, Haines JD, Katz Sand I, Adula KP, Huynh JL, et al. Cerebrospinal fluid ceramides from patients with multiple sclerosis impair neuronal bioenergetics. Brain 2014;137:2271-86.

66. Castle D, Wynford-Thomas R, Loveless S, Bentley E, Howell OW, et al. Using biomarkers to predict clinical outcomes in multiple sclerosis. Pract Neurol 2019;19:342-9. 
67. Chataway J, Schuerer N, Alsanousi A, Chan D, MacManus D, et al. Effect of high-dose simvastatin on brain atrophy and disability in secondary progressive multiple sclerosis (MS-STAT): a randomised, placebo-controlled, phase 2 trial. Lancet 2014;383:2213-21.

68. Kilsdonk ID, Jonkman LE, Klaver R, van Veluw SJ, Zwanenburg JJ, et al. Increased cortical grey matter lesion detection in multiple sclerosis with 7 T MRI: a post-mortem verification study. Brain 2016;139:1472-81.

69. Haider L, Simeonidou C, Steinberger G, Hametner S, Grigoriadis N, et al. Multiple sclerosis deep grey matter: the relation between demyelination, neurodegeneration, inflammation and iron. J Neurol Neurosurg Psychiatry 2014;85:1386-95.

70. Bramow S, Frischer JM, Lassmann H, Koch-Henriksen N, Lucchinetti CF, et al. Demyelination versus remyelination in progressive multiple sclerosis. Brain 2010;133:2983-98. 